\title{
Conducta suicida en Colombia: Una revisión sistemática
}

\author{
Vanessa K. Benavides-Mora, Nixon G. Villota-Melo y Fredy H. Villalobos-Galvis \\ Universidad de Nariño, Colombia
}

\begin{abstract}
Suicide behavior in Colombia: A systematic review. The objective of the review was to synthesize the findings about suicide in Colombia between 2004 and 2018. Eighty-eight studies were included in the review. It is noted that most studies focus on risk factors such as depression, psychoactive substance use and family dysfunction; and only some are interested in protective factors, among which family functionality and high self-esteem are prominent; moreover, the statistical models used are mostly descriptive and studies are not based on theoretical models on suicide. Finally, some lines of research are proposed, such as deepening the theoretical models and statistical analyses used, realizing longitudinal studies and proposing intervention strategies that guide future scientific exercises in the area.
\end{abstract}

Keywords: Suicide; suicidal ideation; suicidal intention; Colombia; systematic review.

Resumen: El objetivo de la revisión fue sintetizar los hallazgos acerca del suicidio en Colombia entre los años 2004 a 2018 . Se incluyeron 88 trabajos en esta revisión. Se destaca que la mayoría de estudios se centran en factores de riesgo como depresión, consumo de sustancias psicoactivas y disfunción familiar; y solo algunos se interesan en factores protectores, entre los que se destacan la funcionalidad familiar y la alta autoestima; además, se encontró que los modelos estadísticos empleados son en su mayoría de tipo descriptivo y los estudios no están basados en modelos teóricos sobre el suicidio. Finalmente, se proponen algunas líneas de trabajo como la profundización de modelos teóricos y análisis estadísticos empleados, la realización de estudios longitudinales y proponer estrategias de intervención, que orienten futuros ejercicios científicos en el área.

Palabras clave: Suicidio; ideación suicida; intención suicida; Colombia; revisión sistemática.

\section{Introducción}

El suicidio es un acto autodestructivo, cuya meta es alcanzar la muerte mediante el método elegido (Baader, Urra, Millán y Yáñez, 2011). Este fenómeno se inicia con la ideación y la intención suicidas hasta llegar al suicidio consumado (Loboa y Morales, 2016). La ideación suicida, está constituida por una variedad de pensamientos, deseos, planes y/o motivaciones de cometer el acto de suicidio (Palacios y Ocampo, 2011; Pérez, Ibáñez, Reyes, Atuesta y Suárez, 2008). Por otra parte, un intento de suicidio es el conjunto de comportamientos iniciados por el propio sujeto, quien al llevarlos a cabo, tiene al menos cierta intención de morir, aunque estos pueden

Recibido: 16 de abril 2019; aceptado: 12 de julio 2019

Correspondencia: Vanessa Katherine Benavides Mora. Universidad de Nariño, Colombia. Correo-e: vanessakbenavidesm@gmail.com causar o no lesiones médicas (American Psychiatric Association, 2013).

El suicidio es un hecho multicausal que no puede atribuirse a un suceso único, razón por la cual, resulta muy relevante el estudio de los factores de riesgo y protectores asociados. Entre los primeros, con mayor frecuencia se destacan: la baja autoestima, la pobreza, el desempleo, trastornos psiquiátricos como la depresión y trastornos del estado de ánimo, la pérdida de un ser querido, situaciones estresantes, conflictos en las relaciones amorosas, la edad, el consumo de sustancias psicoactivas y el maltrato físico y emocional en la infancia, la orientación sexual, entre otros (Cañón y Toro, 2012; Castellví et al, 2017; Kokoulina y Fernandez, 2014; Miranda-Mendizábal et al, 2017; Obando, Trujillo, y Prada, 2018; Sandín et al., 2018; Silva et al., 2017). Incluso, los intentos y pensamientos autodestructivos, que no siempre resultan en la consumación del suicidio, se constituyen también como factores predictores (Castell- 
ví et al, 2017; Palacios y Ocampo, 2010). Por otro lado, como factores protectores se ha destacado la presencia de una adecuada autoestima, altos niveles de habilidad social, apoyo social y familiar (Cañón, 2011; Sánchez-Teruel, Muela-Martínez y García-León, 2018). Sin embargo, no es mucha la literatura que aborda este tipo de factores.

El suicidio se ha convertido en un problema de salud pública mundial, es la segunda causa de muerte en personas con edades entre 15 y 29 años y se afirma que más de 800000 personas se suicidan cada año, siendo los plaguicidas, el ahorcamiento y las armas, los métodos más utilizados. A nivel mundial, los suicidios represen$\tan$ un $50 \%$ de la totalidad de las muertes violentas que se registran entre hombres y un $71 \%$ entre mujeres, sin embargo, dado que en algunos países el suicidio es un acto ilegal es posible que exista una sub-notificación del fenómeno (Organización Mundial de la Salud - OMS, 2017).

En Colombia, se reportaron 19977 suicidios en el decenio 2008-2017, en los 6 primeros años las tasas oscilaron entre 4.2 y 4.5 por 100000 habitantes, mientras que durante el período 2014-2017 han mostrado un ascenso constante de 4.3 a 5.7. Una tendencia similar se ha encontrado en la población de niños, niñas y adolescentes que en el período 2015-2017 presentaron un ascenso en tasas de 3.7 a 4.6, cuando en el período 2008-2014 tuvieron una media de 3.5 ( $D T=0.17$ ) (Montoya, 2018).

Para el año 2017, los grupos etarios con mayor número de suicidios fueron de 20 a 24 y de 25 a 29 , mientras que los de mayores tasas fueron los de 20 a 24 y de 18 a 19. Este comportamiento es similar en hombres y mujeres, aunque en ellas la mayor tasa se da en el grupo de 15 a 17. Igualmente, la mayor proporción de fallecidos por suicidio tenían niveles de escolaridad iguales o inferiores al de secundaria básica y eran solteros. Por su parte, los principales mecanismos causales fueron: generadores de asfixia (64.3\%), sustancias tóxicas $(16.6 \%)$ y arma de fuego (10.9\%), aunque este último predominó principalmente en hombres (Montoya, 2018).

Ante este panorama de salud pública, se encuentra una importante cantidad de estudios empíricos publicados en revistas de libre acceso, en un esfuerzo por describir, comprender e intervenir esta problemática; sin embargo, a la fecha no se conocen cuáles son las tendencias de los estudios científicos del suicidio en Colombia, que permitan determinar los avances e identificar las perspectivas investigativas en esta área del conocimiento. En este sentido, las revisiones sistemáticas permiten tener información eficiente y basada en la evidencia, que resulta muy útil en las ciencias de la salud, pues permiten obtener información de utilidad e identificar tenden- cias dentro de la literatura científica (Oramas y Santaya, 2015; Óscar y Beltrán, 2005; Perestelo-Pérez, 2013).

Al respecto, Salamanca y Siabato (2017) realizaron un análisis de los artículos publicados durante el periodo comprendido entre el 2010 y el 2016 sobre la ideación suicida en población colombiana, encontrando que la temática más abordada en ellos fue la de factores de riesgo y en menor cantidad la de factores protectores, el tipo de investigación predominante es descriptiva y la principal población estudiada es la de estudiantes.

Por otra parte, Vargas y Saavedra (2012) en su revisión sistemática sobre variables asociadas a la conducta suicida en adolescentes, identificaron factores: a) individuales: edad, sexo, etnia, factores biológicos y genéti$\cos$, trastornos mentales, intento suicida previo, orientación sexual, variables relacionadas con la actividad sexual, abuso sexual y físico, y creencias religiosas; b) familiares: conductas suicidas en miembros de la familia, salud física y mental de los miembros de la familia, características socioeconómicas de la familia, estructura familiar y relaciones familiares; y c) sociales: exposición a conductas suicidas de amigos, exposición a medios de comunicación, acontecimientos vitales estresantes, rendimiento escolar, relaciones con pares, soporte social y actividades recreativas, entre otros.

Como se puede observar, las revisiones sobre Conducta Suicida en Colombia se han centrado en un tipo (ideación) o en un solo grupo poblacional (adolescentes), sin embargo, no se cuenta con trabajos que permitan identificar el estado del arte sobre el estudio empírico de las diferentes conductas suicidas en el país, con el fin de proporcionar información acerca del comportamiento del espectro suicida (suicidio consumado, intento, riesgo, ideación), la metodología utilizada, los autores en los que se basan, factores de riesgo y protección comunes e intervenciones realizadas, entre otros.

Teniendo en cuenta lo anterior, el objetivo del presente artículo fue sintetizar los hallazgos acerca del suicidio en Colombia entre los años 2004 a 2018.

\section{Método}

\section{Procedimiento de búsqueda y parámetros de revisión}

Los estudios se seleccionaron de las bases de datos EBSCO, Biblioteca Virtual en Salud (BVS), Scielo, DOAJ, Science Direct, Dialnet y Redalyc, utilizando como palabras clave «Suicid*» en título, resumen o palabras clave; y «Colombia» en texto completo, con el conector «AND».

Los criterios de inclusión para los trabajos fueron: Tener acceso a texto completo, que el estudio se haya 


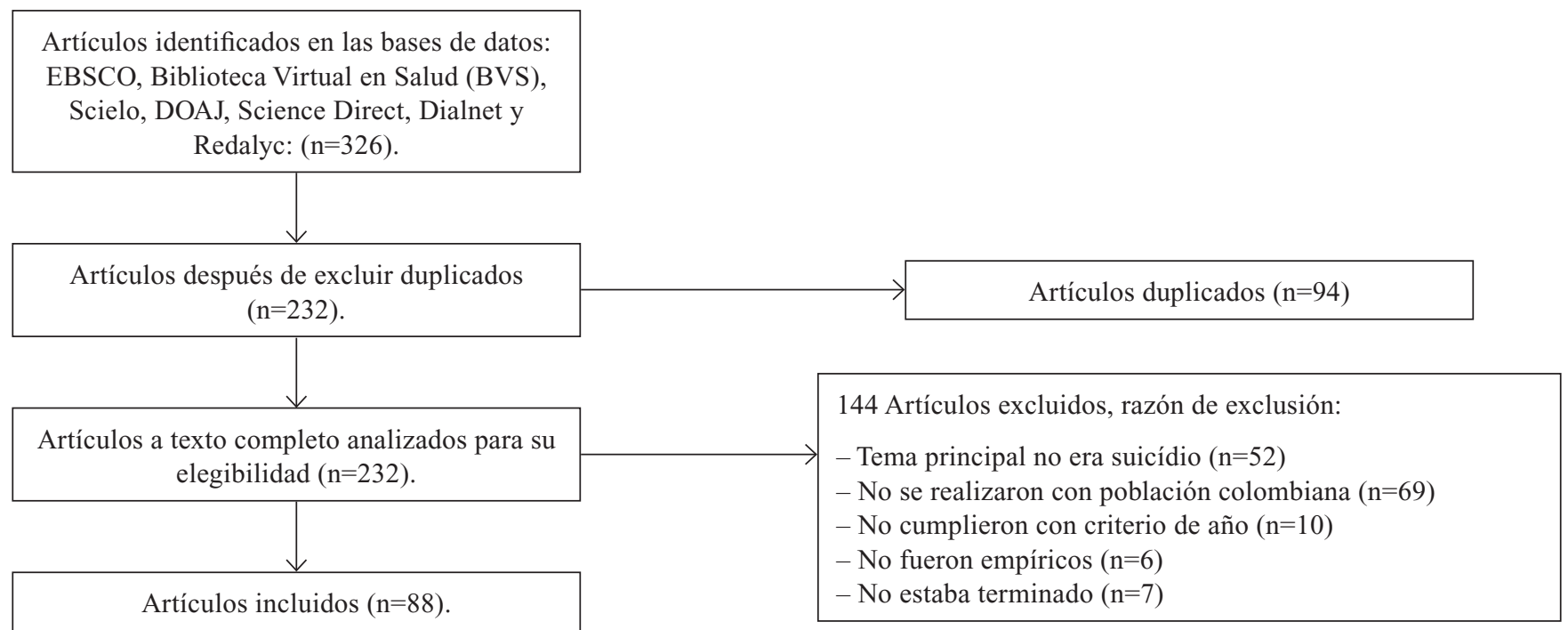

Figura 1. Diagrama de flujo del proceso de selección de estudios (según la guía PRISMA; Moher et al., 2009).

publicado entre los años 2004 a 2018 (se seleccionó este rango de años dado el incremento de conductas suicidas en Colombia durante este periodo), que se haya realizado con población colombiana y que su idioma sea español o inglés. Los criterios de exclusión fueron que el tema principal no sea alguna de las conductas suicidas, que no esté terminado o que no sean estudios empíricos. La búsqueda de información en las bases de datos se llevó a cabo en el período del 15 de diciembre de 2016 a 17 de diciembre de 2018.

\section{Proceso de codificación de los estudios}

Se analizaron las siguientes variables: tipo de conducta, población, técnicas e instrumentos utilizados, tipo de estudio, estadísticos utilizados, autores y teorías en las que se basan los estudios, limitaciones, retos y sugerencias; prevalencia del fenómeno, método utilizado para llevar a cabo la conducta suicida, factores de riesgo, factores protectores y grupos de riesgo. Se realizó un análisis de frecuencias frente a cada una de estas variables.

\section{Resultados $^{1,2}$}

Se identificaron 326 artículos, de los cuales 88 fueron incluidos en la revisión sistemática (véase la Figura 1).

\footnotetext{
1 Todas las citas de resultados se presentarán con el apellido del primer autor seguido de et al. y fecha, con el fin de ofrecer la información al lector sin alterar la longitud del texto de manera significativa.

${ }^{2}$ Las variables que aparecen sin porcentajes corresponden a las encontradas en un solo estudio.
}

\section{Datos generales}

Se identificó que el $87.5 \%$ de los estudios incluidos se desarrollaron durante el periodo de 2010 a 2018, es decir que el estudio sobre suicidio en Colombia se ha incrementado durante los últimos años, en comparación con el periodo comprendido entre 2005 y 2009.

Se observó que solamente 19 estudios $(21.5 \%)$ fueron publicados en revistas internacionales. Por su parte, entre las revistas colombianas se destacan: Revista Colombiana de Psiquiatría, Duazary y Revista de la Facultad Nacional de Salud Pública.

\section{Teoría}

Cuando se revisaron las definiciones de suicidio que son utilizadas en los estudios, se encontró que éstas son principalmente de tipo descriptivo, es decir, se limitan a decir lo que es la conducta suicida, sin embargo, se adolece de definiciones que asuman una postura teórica comprensiva del fenómeno estudiado. Así, la definición más usada es la propuesta por la OMS (11.3\%) que define al suicidio como todo acto en el que un individuo se causa una lesión o daño, con la intención de ocasionar su propia muerte (Calderón-Ramírez, et al., 2017; Cañón-Buitrago, et al., 2017; Ceballos et al., 2012; Dávila-Cervantes, et al., 2017; Fernández-Niño, et al., 2018; García, et al., 2013; Larrotta et al., 2014; Rátiva, et al., 2013; Rodríguez, et al.., 2012; Suescún, et al., 2017) Otra postura es la formulada por Durkheim (10,2\%), quien lo define como un fenómeno social en el que la persona 
atenta contra su vida de forma voluntaria, buscando con ello alcanzar la muerte (Acevedo, 2010; Andrade et al., 2010; Andrade et al., 2013; Chaparro-Narváez, 2018; Franco, et al., 2017; Jaramillo et al., 2015; Pérez, 2014; Sepúlveda, 2008; Suarez, et al., 2016; Suarez, et al., 2017). Finalmente, otros estudios retoman la Clasificación Internacional de Enfermedades (CIE11) $(4.5 \%)$ según la cual el suicidio se define como la muerte por lesión autoinfligida y deliberadamente iniciada por la persona (Arenas, et al., 2016; Toro-Tobar, et al., 2016, Velásquez, et al., 2017). Otras teorías o autores que definen el suicidio, solo se utilizan en uno o dos estudios, por su parte algunos estudios no definen este fenómeno.

\section{Metodología de los estudios}

Se organizaron los tipos de estudio de acuerdo con el paradigma, alcance, temporalidad (Hernández, Fernández y Baptista, 2010; Montero y León, 2002) e investigación clínica (Manterola y Otzen, 2014; Pardo y Cedeño, 1997), sin embargo, no todos los estudios mencionan alcance y temporalidad.

Se encontraron estudios con paradigma cuantitativo $(82.9 \%)$, los cuales se caracterizan por ser rigurosos y secuenciales, en estos se establecen hipótesis y determi- nan variables, las cuales se comprueban con un diseño de investigación establecido. Se realizan mediciones estadísticas y análisis de dichas variables para finalmente extraer una serie de conclusiones. Por su parte, se encontraron pocos estudios cualitativos (10.2\%) (ver Tabla 1 ), en los cuales se pueden desarrollar preguntas e hipótesis antes, durante o después de la recolección y el análisis de los datos. La acción indagatoria resulta un proceso más bien «circular» en el que la secuencia no siempre es la misma, pues varía con cada estudio (Hernández, Fernández y Baptista, 2010). También se encontraron revisiones sistemáticas $(4.5 \%)$ y dos estudios que combinaron estrategias cuantitativas y cualitativas $(2.2 \%)$. Solo un estudio cualitativo menciona temporalidad (retrospectivo).

Finalmente, un estudio realizado bajo los paradigmas cualitativo y cuantitativo menciona tener alcance descriptivo $(50 \%)$.

\section{Población}

En cuanto a la población objeto de estudio, los resultados se clasificaron según el grupo etario, ocupación, condición y ubicación geográfica (ver Tabla 2). Se debe considerar que 18 estudios no se realizaron con población directa por ser revisiones de estadísticas oficiales e

Tabla 1. Tipos de estudio

\begin{tabular}{|c|c|c|c|}
\hline \multicolumn{2}{|c|}{ Paradigma Cuantitativo } & \multicolumn{2}{|c|}{ Paradigma Cualitativo } \\
\hline Alcance & $\mathrm{N}^{\mathrm{o}}$ de estudios & Alcance & $\mathrm{N}^{\mathrm{o}}$ de estudios \\
\hline Descriptivos & $26(35.6 \%)$ & Hermenéutico & $5(55.5 \%)$ \\
\hline Correlacional & $18(24.6 \%)$ & Descriptivos & $2(22.2 \%)$ \\
\hline Instrumental & $4(5.4 \%)$ & Fenomenológico & $2(22.2 \%)$ \\
\hline Comparativo & $4(5.4 \%)$ & Estudio de caso & $2(22.2 \%)$ \\
\hline Cuasi experimental & $1(1.3 \%)$ & Interaccionismo simbólico & $1(11.1 \%)$ \\
\hline Temporalidad & $\mathrm{N}^{\circ}$ de estudios & Etnográfico & $1(11.1 \%)$ \\
\hline Transversal & $28(38.3 \%)$ & Proyecto de intervención & $1(11.1 \%)$ \\
\hline Retrospectivo & $9(12.3 \%)$ & & \\
\hline Longitudinal & $4(5.4 \%)$ & & \\
\hline Prospectivo & $2(2.7 \%)$ & & \\
\hline Investigación clínica & $\mathrm{N}^{\mathrm{o}}$ de estudios & & \\
\hline Casos y controles & $5(5.6 \%)$ & & \\
\hline Ecológico & $5(5.6 \%)$ & & \\
\hline Observacional analítico & $3(3.4 \%)$ & & \\
\hline Observacional descriptivo & $3(3.4 \%)$ & & \\
\hline Analítico & $3(3.4 \%)$ & & \\
\hline Observacional & $2(2.2 \%)$ & & \\
\hline
\end{tabular}


institucionales o revisiones sistemáticas. Además, llama la atención la existencia de varios estudios que no reportan, en la sección de método, los datos de la población con la cual trabajaron, sino que lo hacen como parte de los resultados.

Tabla 2. Población objeto de estudio

\begin{tabular}{|c|c|c|c|}
\hline \multicolumn{4}{|c|}{ Población } \\
\hline & & $N$ & $\%$ \\
\hline \multirow[t]{5}{*}{ Grupo etario } & Niños & 3 & $3.4 \%$ \\
\hline & Adolescentes & 23 & $26.1 \%$ \\
\hline & Jóvenes & 10 & $11.3 \%$ \\
\hline & Adultos & 6 & $7 \%$ \\
\hline & No menciona & 32 & $36.3 \%$ \\
\hline \multirow[t]{6}{*}{ Ocupación } & Estudiantes primaria & 4 & $4.5 \%$ \\
\hline & Estudiantes secundaria & 15 & $17 \%$ \\
\hline & Estudiantes universitarios & 11 & $12.5 \%$ \\
\hline & Policías & 2 & $2.2 \%$ \\
\hline & Agentes universitarios & 2 & $2.2 \%$ \\
\hline & No menciona & 41 & $46.5 \%$ \\
\hline \multirow[t]{6}{*}{ Condición } & Líderes sociales & 1 & $1.1 \%$ \\
\hline & Consumaron suicidio & 1 & $1.1 \%$ \\
\hline & Reclusos & 3 & $3.4 \%$ \\
\hline & Familiares & 4 & $4.5 \%$ \\
\hline & Pacientes & 18 & $20.5 \%$ \\
\hline & No menciona & 39 & $44.3 \%$ \\
\hline \multirow{2}{*}{$\begin{array}{l}\text { Ubicación } \\
\text { geográfica }\end{array}$} & Nacional & 3 & $3.4 \%$ \\
\hline & Departamental & 67 & $76.1 \%$ \\
\hline
\end{tabular}

\section{Técnicas e instrumentos}

Dentro de las técnicas de recolección de información sobresalieron la revisión de estadísticas oficiales e institucionales (27\%) (Arroyave, et al., 2007; Bilbao, et al., 2016; Brito, et al., 2011; Campo-Arias, et al., 2014; Daza, et al., 2011; Fernández-Niño, et al., 2018; Guerrero-Martínez, 2016; Medina, et al., 2010; Medina-Pérez, et al., 2017; Ortega, et al., 2014; Ortegón, et al., 2018; Peña, et al., 2009; Rodríguez-Hernández, et al., 2018; Sánchez, et al., 2004), las entrevistas (16\%) (Ceballos, 2004; Palacios, et al., 2006; Rueda, et al., 2010; Rueda et al., 2011), la autopsia psicológica (2.2\%) (Muñoz, et al., 2010), los cuestionarios creados específicamente para cada estudio (2.2\%) (Gonzales-Portillo, et al., 2016; Palacio, et al., 2005; Rueda et al., 2011) y el Formato único de regis- tro para casos de comportamientos suicidas (Larrota, et al., 2014).

Por su parte, los instrumentos psicométricos más utilizados para la medición y evaluación de la conducta suicida fueron: el Inventario de Ideación Suicida Positiva y Negativa (PANSI) (11.3\%) cuyo objetivo es evaluar factores de riesgo y protección frente a las ideas de suicidio (Luna, et al., 2017; Martínez, et al., 2017; Moreno-Montoya, et al., 2017; Pérez, 2012 et al., Quiceno, et al, 2013; Quiceno, et al., 2013; Siabato, et al., 2016; Villalobos, 2009), el Cuestionario de Plutchick (9\%), el cual permite diferenciar entre individuos que no presentan riesgo suicida y los que sí están en él (Aguirre et al., 2015; Álvarez et al., 2013; Amaya et al., 2013; Bahamón, et al., 2018; Cañón et al., 2012; Cañón et al., 2012; Pereira-Morales, et al., 2017), y el Inventario de Orientación Suicida (ISO) (4.5\%), utilizado para el despistaje de la presencia de riesgo suicida (Gonzales, et al., 2016; Paniagua, et al., 2014; Reyes, et al., 2005; Rodríguez, et al., 2013). Otros instrumentos usados en menor proporción fueron: la Cédula de Indicadores Parasuicidas (CIP), el cual permite conocer la ocurrencia de intentos suicidas y el número de estos a lo largo de la vida, la edad del único o último intento, los motivos y métodos (Villalobos, 2009); el Inventario de Resiliencia ante el Suicidio (IRS-25), diseñado para medir los factores que ayudan a preservar de pensamientos y conductas suicidas (Villalobos, et al., 2012) y el Cuestionario de Ideación Suicida (SIQ) cuyo objetivo es evaluar la frecuencia de una jerarquía de pensamientos suicidas, partiendo desde los posibles pensamientos de desear no estar vivo, hasta pensamientos serios y específicos de matarse a sí mismo (Villalobos, et al., 2012). A excepción del cuestionario de Plutchik, los instrumentos anteriormente mencionados han sido validados en Colombia. Resulta importante mencionar que un estudio resalta la utilidad de la autopsia psicológica (Andrade, et al., 2013), como un instrumento que recoge datos acerca del occiso para reconstruir su perfil psicológico y su estado mental antes de la muerte, a partir de entrevistas a familiares, amigos y personas cercanas a la persona fallecida y otros procesos de investigación, lo anterior con el fin de esclarecer los casos en los que la muerte por suicidio es dudosa (Rodríguez, 2001).

\section{Análisis de datos cuantitativo}

Se describieron los estadísticos utilizados para el análisis de datos en los diferentes estudios revisados y fueron agrupados de acuerdo a la finalidad de los mismos como se muestra en la Tabla 3. 
Tabla 3. Tipos de estadísticas

\begin{tabular}{lcc}
\hline \multicolumn{1}{c}{ Tipo de estadísticas } & $\begin{array}{c}\text { Número } \\
\text { de estudios }\end{array}$ & \% \\
\hline Descriptivas & 59 & $67 \%$ \\
Bondad de ajuste/comprobación & 11 & $12.5 \%$ \\
de supuestos & & \\
Inferencial & & \\
$\quad$ Correlación/asociación & 39 & $44.3 \%$ \\
$\quad$ Diferencia/conformación de grupos & 22 & $25 \%$ \\
$\quad$ Predicción & 19 & $21.6 \%$ \\
Epidemiológicas & 8 & $9.1 \%$ \\
Análisis psicométricos & 8 & $9.1 \%$ \\
\hline
\end{tabular}

\section{Análisis de datos cualitativo}

Dentro de las técnicas de análisis cualitativo utilizadas en los estudios revisados se encontraron: Categorización (9.1\%) (Franco, et al., 2017; Ojeda, et al., 2011), en menor medida (2.3\%) análisis narrativo (Rendón-Quintero, et al., 2016; Urrego-Mendoza, et al., 2017) y construcción de tendencias (Builes, 2014; Manco, 2015), y se encontraron en un solo estudio la lectura interpretativa de las dimensiones sociales y subjetivas del fenómeno (Pérez, 2014), condensación hermenéutica (Barrios-Acosta, et al., 2017) y análisis de estructura y contenido (Daza, et al., 2007); cabe resaltar que esta última es una técnica ad hoc creada para realizar un análisis de noticias.

\section{Tipo de conducta estudiada}

El suicidio consumado fue el tipo de conducta suicida más investigado, seguida de la ideación suicida y del intento (ver Figura 2).

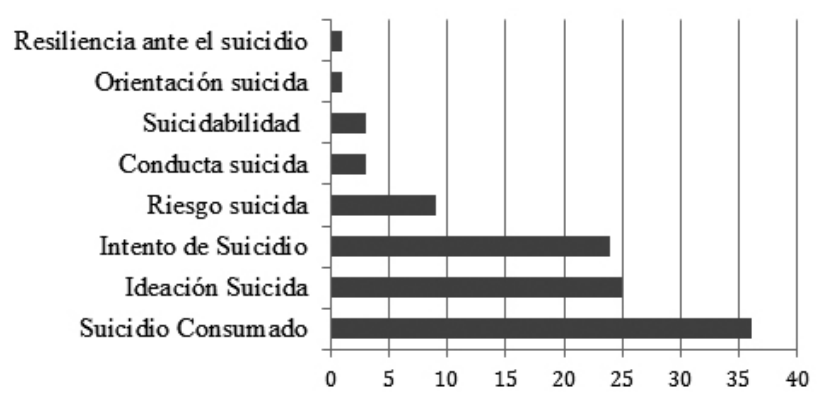

Figura 2. Tipos de conducta suicida estudiados (número de estudios que incluyen cada tipo).

\section{Factores de riesgo y protección identificados}

Se evidenció que en los estudios prevalece la información acerca de factores de riesgo, encontrando así que 57 de ellos (65\%) presentaron resultados sobre este tipo de variables, las cuales pueden ser organizadas en 7 grupos de factores: a) Salud: depresión (21.5\%), diagnóstico psiquiátrico (18\%), consumo de sustancias psicoactivas $(17 \%)$, intentos previos de suicidio $(10 \%)$ y enfermedad biológica (8\%); b) Familiares: disfunción familiar (20\%), antecedentes familiares de suicidio $(8 \%)$ y bajo apoyo familiar $(4.5 \%)$; c) Sociodemográficos y económicos: vivir solo/soltero (11.3\%), bajos ingresos $(11.3 \%)$, baja escolaridad $(7 \%)$, acceso a mecanismos (6\%), desempleo (3.4\%), deshumanización de la educación y menores variaciones en el crecimiento anual del PIB per cápita; d) Interpersonales: conflictos en relaciones interpersonales o de pareja (11.3\%), aislamiento (4.5\%) y bajo apoyo social; e) Estresores: víctima de abuso sexual (10\%), víctima de maltrato/violencia (9\%), pérdida de ser querido $(7 \%)$, eventos estresantes (6\%), víctima de acoso o intimidación (3.4\%) y víctima de desplazamiento forzado o conflicto armado (2.2\%); y f) Personalidad: baja autoestima $(8 \%)$, desesperanza $(4.5 \%)$ y dificultades para tolerar la frustración $(3.4 \%)$.

Por otra parte se identificó que fue reducido el número de investigaciones que exponen resultados acerca de factores protectores, encontrando 26 estudios (29.5\%) que plantean los siguientes grandes factores: a) Familia: Funcionalidad familiar $(11.3 \%)$; b) Personalidad: alta autoestima (7\%); estado de ánimo positivo, regulación e inteligencia emocionales $(6 \%)$, resiliencia $(3.4 \%)$, razones para vivir $(2.2 \%)$, estabilidad del vínculo afectivo (2.2\%), Estrategias de supervivencia y afrontamiento $(2.2 \%)$ e ideas positivas frente al futuro; c) Sociodemográficos: Pertenecer a una religión $(7 \%)$, mayor edad $(2.2 \%)$, mayores variaciones en el crecimiento anual del PIB per cápita, tener un trabajo formal, alto estrato económico, características culturales particulares de las subregiones; d) Interpersonales: Apoyo social y familiar $(6 \%)$, redes que promueven conductas saludables, tener una relación de pareja estable; e) Relacionadas con el suicidio: miedo a fracasar en el intento, objeciones morales, miedo a la desaprobación social a las personas que tienen conductas suicidas, miedo al suicidio y difícil acceso al método de suicidio; f) Servicios de salud y bienestar: bienestar físico, práctica de actividades lúdicas y deportivas, bienestar psicológico de calidad de vida, haber asistido a consultas psicológicas o psiquiátricas, implementación de programas que promueven habilidades y competencias personales, pertenecer a grupos artísticos o cívico comunitarios; y g) Educación: mejor calidad (2.2\%), enseñanza de normas, deberes, principios éticos y derechos. 


\section{Mecanismo utilizado}

Dentro de los estudios que abordan la suicidabilidad, los intentos y los suicidios consumados, los principales mecanismos hallados son los generadores de asfixia, los tóxicos y los cortopunzantes (ver Tabla 4).

Tabla 4. Mecanismos utilizados para llevar a cabo la conducta suicida

\begin{tabular}{|c|c|c|c|c|c|c|c|}
\hline \multirow{2}{*}{ Mecanismo } & \multirow{2}{*}{ Método } & \multicolumn{2}{|c|}{ Suicidio } & \multicolumn{2}{|c|}{ Intento de Suicidio } & \multicolumn{2}{|c|}{ Suicidabilidad } \\
\hline & & $N$ & $\%$ & $N$ & $\%$ & $N$ & $\%$ \\
\hline \multirow{2}{*}{ Generadores de Asfixia } & Ahorcamiento & 18 & $62 \%$ & 4 & $14 \%$ & 3 & $10.3 \%$ \\
\hline & Ahogamiento & 2 & $7 \%$ & 1 & $3.4 \%$ & - & - \\
\hline \multirow{3}{*}{ Tóxicos } & Intoxicación & 7 & $24 \%$ & 4 & $14 \%$ & 3 & $10.3 \%$ \\
\hline & Envenenamiento & 9 & $31 \%$ & 4 & $14 \%$ & - & - \\
\hline & Sobredosis & 4 & $14 \%$ & 4 & $14 \%$ & 3 & $10.3 \%$ \\
\hline Armas cortopunzantes & Armas cortopunzantes & 10 & $34.4 \%$ & 6 & $21 \%$ & 2 & $7.0 \%$ \\
\hline Armas de fuego & Armas de fuego & 11 & $38 \%$ & 1 & $3.4 \%$ & 1 & $3.4 \%$ \\
\hline Contundente & Lanzamiento al vacío & 7 & $24 \%$ & - & - & 3 & $10.3 \%$ \\
\hline \multirow{2}{*}{ Otros } & Accidente automotor & - & - & 1 & $3.4 \%$ & 3 & $10.3 \%$ \\
\hline & Otros (incendiarse a sí mismo, inyectarse aire) & 4 & $14 \%$ & 1 & $3.4 \%$ & - & - \\
\hline
\end{tabular}

\section{Propuestas de intervención}

De los estudios revisados, solamente el $4.5 \%$ de ellos plantearon propuestas, modelos o estrategias de intervención frente al suicidio: a) un modelo de intervención en crisis basado en el interaccionismo simbólico, en el cual se identificaron dos tipos de impulsos frente al suicidio, estos a su vez se dividen en dos subtipos respectivamente, los cuales fueron utilizados como base para establecer estrategias de intervención (Jaramillo et al., 2015); b) un modelo de intervención comunitaria, en el cual se plantea la utilización de métodos terapéuticos que permitan trabajar de manera conjunta con comunidades indígenas, respetando su cosmovisión y sin apartarlos de su lugar de vivienda (Sepúlveda, 2008); c) propuestas institucionales en el contexto universitario, que incluían identificación e intervención de factores de riesgo, promoción de hábitos de vida saludables, y estrategias de educación y sensibilización frente a vulnerabilidad psicológica, estrés académico y cómo llevar a cabo el primer contacto con estudiantes con riesgo suicida (Barrios-Acosta, et al., 2017) y d) una propuesta de intervención educativa para adolescentes, en la cual se desarrollaron actividades en torno a la autoestima y la comunicación asertiva a través del arte y expresión corporal (Cañón et al., 2018).

Es interesante resaltar que sólo un estudio de los revisados estableció un modelo teórico como base de una propuesta de intervención en crisis para niños y adolescentes (Jaramillo, et al., 2015). Se proponen dos tipos de impulso al suicidio, los cuales son: Tipo anómico. Caracterizado por sentimientos de soledad, deficientes redes de apoyo y ausencia de reglas, por lo cual se convierte en un llamado a la ayuda, atención y acompañamiento, además, se asocia con síntomas depresivos, inseguridad y baja autoestima. Este tipo de divide en el subtipo ambivalente, en el que predomina la ausencia de reglas o límites claros frente a las conductas de los niños y adolescentes; y el subtipo desesperado, caracterizado por la ausencia o deficiencia de redes de apoyo. El segundo es el Tipo exaltado. El niño o adolescente se encuentra en un contexto hostil, dentro del cual no encuentra la manera de aprender a solucionar los conflictos mediante el diálogo y toma posiciones de confrontación o agresión, y en algunos casos se presenta el consumo de sustancias psicoactivas, se asocia con la proyección como mecanismo de defensa, baja tolerancia a la frustración y la dificultad en la autorregulación y control de impulsos. Se divide en los subtipos «fosforito» en el cual el impulso se presenta después de una situación adversa como una discusión o un castigo; y el subtipo «fosforito exaltado», el cual se caracteriza por la respuesta impulsiva a situaciones problemáticas, mediada por estados alterados de conciencia debido al consumo de sustancia psicoactivas.

\section{Grupos de riesgo}

A lo largo de los diferentes estudios, se encontraron como principales grupos de riesgo los adultos jóvenes 
(23\%), adolescentes (17\%), mujeres (15\%), hombres $(18 \%)$, personas con estado civil soltero $(4.5 \%)$, pacientes con trastorno mental (2.2\%), adultos mayores de cuarenta años y comunidad indígena expuesta al conflicto armado.

\section{Prevalencias}

El estudio de prevalencias de las conductas suicidas arrojó datos según el tipo de conducta analizado. Frente al riesgo suicida, la prevalencia en adolescentes fue de $16.5 \%$ (Aguirre et al., 2015), mientras que en estudiantes universitarios fue de $13.5 \%$ (Cañón et al., 2012), en pacientes de consulta externa fue de 39\% (Amaya et al., 2013) y en mujeres privadas de la libertad fue de $14.4 \%$ (Cañón, et al., 2016). En cuanto a orientación suicida, en adolescentes la prevalencia fue de $11.3 \%$ (Paniagua et al., 2014).

En lo correspondiente a ideación suicida se encontraron varios estudios en adolescentes en los cuales la prevalencia osciló entre el 5.1\% y el 30\% (Carvajal, et al., 2011; Ceballos et al., 2012; Forero, et al., 2017; Molina et al., 2011; Pérez, 2014; Piedrahita et al., 2012; Villalobos, 2010). También se encontraron estudios en pacientes de Instituciones Prestadoras de Salud, con prevalencias de $25 \%$ (Amaya et al., 2013) y 49.9\% (Rodríguez et al., 2012).

Con respecto al intento de suicidio, se halló una prevalencia de $2.9 \%$ en una muestra de policías (Rodríguez et al., 2013), 8\% en adolescentes escolarizados (Villalobos, 2010) y $15 \%$ en adolescentes que habían sufrido abuso sexual (Pérez, et al., 2017).

\section{Limitaciones reportadas}

Las principales limitaciones comentadas en los diferentes estudios estuvieron relacionadas con el tamaño de muestra (9\%), tipo de diseño (7\%), sesgo en las respuestas $(6 \%)$; tipo de población, casos de no respuesta al instrumento, técnica utilizada y dificultad para acceder a los datos (3.4\%); pérdida de participantes durante el proceso investigativo $(2.2 \%)$, instrumentos no validados, sustento teórico, sub-reporte de casos y escaso conocimiento del proceso investigativo por parte de los participantes.

\section{Retos y sugerencias formuladas}

Con respecto a los retos o sugerencias presentados en los estudios, llama principalmente la atención la necesidad de acciones de promoción de la salud mental y de prevención de las conductas suicidas (19.3\%), darle con- tinuidad y replicar los estudios en muestras más amplias y diferentes contextos $(10 \%)$, realizar trabajos con diferentes tipos de estudio como epidemiológicos, explicativos y con diseños longitudinales (3.4\%), ahondar en variables como: variables relacionadas con la familia; variables psicológicas como depresión, ansiedad o trastornos alimenticios; elementos socioculturales y representaciones sociales; y eventos adversos o situaciones vitales estresantes como el abuso sexual, enfermedades crónicas, bullying, fracaso escolar, contexto universitario y la transición a la vida laboral $(3.4 \%)$, validación de estrategias de intervención (2.2\%), validar instrumentos de medición y generar campañas para la adecuada difusión de noticias de suicidio a través de medios de comunicación.

\section{Discusión}

El objetivo del presente estudio fue sintetizar los hallazgos acerca del suicidio en Colombia entre los años 2004 a 2018, este propósito resulta pertinente en la medida en que el suicidio es catalogado como un problema de salud pública a nivel internacional (OMS, 2014). En este punto se encuentra relación entre el aumento de tasas de suicidio y el incremento de publicaciones sobre el tema en los últimos 10 años, demostrando una congruencia entre el interés público y el interés académico, no obstante inquieta que a pesar de ello, las tasas de mortalidad por suicidio vienen en un incremento continuo desde el año 2014, por lo cual se requiere de mayores aportes orientados al cambio de esta tendencia.

En cuanto a los resultados, sobre la teoría en la que se basan los distintos estudios revisados, se puede observar que se trabajó en su mayoría con las definiciones de Durkheim y de la OMS, por lo cual se puede afirmar que en Colombia se están utilizando teorías descriptivas frente al suicidio, lo cual contrasta con lo propuesto por Jiménez (2011) quien señala la importancia de trabajar con teorías y modelos explicativos frente a este fenómeno, de tal manera que se logre no solo su descripción, sino una mejor comprensión, análisis e intervención. En este sentido, se resalta la propuesta teórica de un estudio cualitativo en el que se buscó establecer una propuesta de intervención en niños y adolescentes (Jaramillo, et al., 2015). Por esto, resulta importante fomentar el desarrollo y la validación de modelos teóricos, que ofrezcan comprensiones profundas y complejas del fenómeno del suicidio y abran nuevas perspectivas para su pertinente intervención.

Los tipos de estudio más comunes en los trabajos revisados fueron los cuantitativos, con alcance descriptivo y correlacional, mientras que se encontraron pocos estu- 
dios cualitativos, los cuales proporcionan una mirada integral del ser humano (Vanegas, 2011), sin embargo, se presenta dificultad para integrar sus resultados ideográficos a propuestas de salud pública. Frente a este punto, también es necesario realizar estudios instrumentales que permitan adaptar o crear instrumentos, teniendo en cuenta la importancia de contar con certezas frente a la validez y confiabilidad de los datos obtenidos en los estudios (Carretero-Dios y Pérez, 2007).

Por su parte, los estudios se realizaron con una temporalidad transversal, por lo cual se observa la necesidad de realizar estudios longitudinales sobre el suicidio en Colombia, ya que como lo refieren Pérez y Martín (2004) este tipo de estudios permiten realizar un seguimiento sistemático a las variables estudiadas a través del tiempo en los mismos sujetos, de este modo se puede identificar la causalidad de una determinada condición de salud o enfermedad.

En coherencia con lo anterior, es preponderante el uso de estadísticas descriptivas, las cuales se emplearon en todos los estudios cuantitativos. Se encontró un vacío importante frente al establecimiento de modelos estadísticos que permitan dar una mejor definición metodológica al estudio del suicidio en Colombia, ya que es escaso el uso de métodos estadísticos más avanzados, como los métodos multivariados, situación acorde con lo encontrado por Massip, Soler y Torres (2011) y Pérez (2010) quienes en sus estudios muestran que en ciencias de la salud y psicología, se utilizan en su mayoría estadísticos descriptivos. Teniendo en cuenta lo anterior, se recomienda hacer uso de modelos multivariados que aporten datos para la identificación y comprensión multifactorial de factores de riesgo y protección, que faciliten la comprobación empírica de modelos teóricos multicausales frente al suicidio y aporten al establecimiento de propuestas de prevención e intervención del fenómeno (Grisales, 2006).

También, en coherencia con lo propuesto por Argibay (2009), se recomienda ampliar el tamaño y diversificar la composición de las muestras de estudio, incluyendo diferentes grupos poblacionales, de tal forma que se cuente con una mirada colectiva que ofrezca elementos para la formulación de políticas públicas.

En lo que corresponde a la población, se identificó que la mayoría de estudios se realizaron con adolescentes y jóvenes estudiantes, esto es acorde con cifras nacionales encontradas en los reportes del Instituto de Medicina Legal y Ciencias Forenses las cuales evidencian un aumento en la prevalencia de conductas suicidas en poblaciones jóvenes en los últimos diez años (Macana, 2011; Montoya, 2018), de igual forma, según la OMS (2017) la prevalencia del suicidio es mayor en las perso- nas de 15 a 29 años. Es importante mencionar que las tasas de suicidio también son elevadas entre grupos vulnerables (OMS, 2018), sin embargo, sólo uno de los estudios incluidos en la presente revisión se realizó con población indígena y dos de ellos con personas privadas de la libertad. Además, una alta cantidad de estudios no describieron las características de la población con la que trabajaron en el apartado de metodología, si no que las enunciaron como parte de sus resultados, frente a lo cual es importante resaltar que, como lo propone Cantú (2015) es en el método en donde se deben incluir los datos de la población y muestra, así como sus criterios de inclusión y exclusión, ya que este apartado garantiza la validez y rigurosidad científica de los estudios.

En cuanto a instrumentos, si bien encontraron algunos que permiten medir y evaluar diferentes atributos relacionados con el suicidio en Colombia, es importante darle continuidad a los estudios instrumentales que permitan tener instrumentos validados en el país y en poblaciones específicas, para una valoración válida y confiable del fenómeno en cuestión, puesto que el proceso de validación de instrumentos, así como el reporte de sus propiedades psicométricas son elementos indispensables en todo proceso de medición dentro de la investigación científica y en la toma efectiva de decisiones en base a dichas mediciones (Soriano, 2014).

En consonancia con los hallazgos de Salamanca y Siabato (2017), en la presente revisión sistemática se evidenció que la mayoría de estudios se centraron en factores de riesgo y son limitados aquellos que abordan la temática de factores protectores frente al suicidio. Se resalta la necesidad de estudiar los grupos de factores de riesgo, con el fin de plantear propuestas de prevención. Por su parte, la identificación de factores protectores permitirá un cambio de paradigma de intervención en el que no solamente se controlen los factores patogénicos, sino que se formulen programas y acciones de promoción de la salud mental (Amar, Abello, y Acosta, 2003).

Entre los mecanismos de suicidio más reportados en los estudios se destacaron los generadores de asfixia, tóxicos y armas cortopunzantes, lo cual guarda concordancia con datos nacionales como los del Instituto de Medicina Legal y Ciencias Forenses (Macana, 2011) y el Ministerio de Salud y Protección Social (2017). Estos datos reafirman la importancia de las políticas públicas centradas en el control de la disponibilidad del método que se han implementado a nivel mundial (Jenkins y Singh, 2000), no obstante, se requieren nuevas miradas sobre el problema, orientadas a la prevención de otros métodos, considerados de fácil acceso en distintos contextos.

En lo concerniente a modelos de intervención propuestos, sólo 4 de los trabajos revisados formularon una 
propuesta, lo cual muestra un vacío frente a la forma de abordar el fenómeno del suicidio en Colombia, convirtiéndose en un reto para los investigadores llegar a una comprensión más profunda del suicidio, con el fin de que pueda ser intervenido y no solo descrito, ya que como lo plantea Aranguren (2009), el suicidio es un fenómeno de difícil comprensión y tiene que ser analizado desde distintas perspectivas como la psicológica, biológica o sociológica, y resulta indispensable apoyarse en modelos teóricos que permitan obtener resultados positivos en su intervención.

Al tener en cuenta las limitaciones, retos y sugerencias de los estudios revisados, así como lo expuesto anteriormente, se formulan las siguientes recomendaciones para futuras investigaciones sobre conductas suicidas en Colombia:

El primer reto para los investigadores en Colombia consiste en profundizar en distintas teorías que permitan un mejor análisis de las conductas suicidas o formular modelos teóricos que respondan a las condiciones particulares del suicidio en el medio local y nacional colombiano y que orienten el abordaje científico y/o profesional del suicidio como un fenómeno humano. Un ejemplo es el modelo estrés-afrontamiento propuesto por Lazarus y Folkman a partir del cual se pueden proponer estrategias de prevención y promoción frente al suicidio (Villalobos, 2006). Además, la formulación de modelos teóricos posibilita la realización de estudios que formulen, sistematicen y evalúen formas de abordaje de las conductas suicidas, de cara a la generación de intervenciones con apoyo empírico que faciliten y masifiquen su implementación por parte de profesionales de la salud mental.

Por otro lado, hay una gran necesidad de estudios longitudinales que posibiliten un acercamiento a la «historia natural» de las conductas suicidas, de tal manera que ofrezcan elementos de diagnóstico e intervención temprana en contextos de salud pública (Pérez y Martín, 2004).

Finalmente, es necesario recordar que el suicidio es un fenómeno de graves implicaciones en la vida de las personas, las comunidades y la sociedad general, situación frente a la cual la investigación científica debe posicionarse con mayor pertinencia, de cara a ofrecer a la sociedad elementos que permitan un manejo idóneo. La presencia de modelos teóricos, metodológicos y/o estadísticos más complejos permitirá una mejor comprensión del espectro suicida en Colombia, desde el cual se hará factible la formulación de programas de intervención orientados a la promoción de la salud mental y la prevención del fenómeno, en pro del mejoramiento de las condiciones de vida de las personas y las comunidades del país.
Una de las limitaciones que tuvo la presente revisión fue que se tomaron solamente artículos científicos, por lo cual no se revisaron trabajos no publicados en revistas científicas, como tesis de pregrado o postgrado que traten sobre el tema. Por otra parte, se consultaron bases de acceso abierto, dejando de lado la información de estudios de bases de acceso restringido.

\section{Referencias}

Acevedo, C. (2010). ¿Influye la realidad económica en el suicidio? El caso colombiano. Economía del Caribe, 5, 143-87.

Aguirre, D., Castaño, J., Cañón, S., Sánchez, D., Rodríguez, J., Rosero, L., ... y Veléz, J. (2015). Riesgo suicida y factores asociados en adolescentes de tres colegios de la ciudad de Manizales (Colombia), 2013. Revista de la Facultad de Medicina, 63(3), 419. DOI: 10.15446/revfacmed.v63n3.44205

Álvarez, J., Cañón, S., Castaño, J., Berner, L., Cataño, A., Galdino, P., ... y Sánchez, C. (2013). Factor de riesgo suicida y factores asociados en adolescentes de una institución educativa de Palestina-Caldas (Colombia), 2012. Archivos de Medicina (Col), 13(2), 127-141. DOI: 10.30554/archmed.13.2.153.2013

Amar, J., Abello, R., y Acosta, C. (2003). Factores protectores: Un aporte investigativo desde la psicología comunitaria de la salud. Psicología desde el Caribe, 11, 107-121.

Amaya, A., Bruce, A., Herrán, D., Martín, A., Muñoz, K., y Abella, P. (2013). Variables asociadas a riesgo de suicidio en pacientes con dolor crónico atendidos por consulta externa en un hospital de Bogotá. Revista Colombiana de Anestesiología, 41(4), 267-273. DOI: 10.1016/j.rca.2013.06.007

American Psychiatric Association (APA). (2013). Diagnostic and statistical manual of mental disorders $\left(5^{\text {th }}\right.$ ed.). Washington, DC: APA.

Andrade, J., Bonilla, L., y Valencia, Z. (2010). Factores protectores de la ideación suicida en 50 mujeres del Centro Penitenciario: «Villa Cristina»-Armenia-Quindío (Colombia). Orbis. Revista Cientifica Ciencias Humanas, 17, 6-32.

Andrade, J., Téllez, C., y Giraldo, L. (2013). La Autopsia Psicológica en Colombia: Exploración Psicobiográfica del Suicidio. Revista Internacional de Psicología, 12(2). DOI: 10.33670/18181023.v12i02.70

Aranguren, M. (2009). Modelos teóricos de comprensión del Suicidio. I Congreso Internacional de Investigación y Práctica Profesional en Psicología. XVI Jornadas de Investigación Quinto Encuentro de Investigadores en Psicología del MERCOSUR. Buenos Aires, Argentina.

Arenas, A., Gómez-Restrepo, C., y Rondón, M. (2016). Factores asociados a la conducta suicida en Colombia. Resultados de la Encuesta Nacional de Salud Mental 2015. Revista Colombiana de Psiquiatría, 45(1), 68-75. DOI: 10.1016/j.rcp.2016.03.006 0034-7450

Argibay, J. (2009). Muestra en investigación cuantitativa. Subjetividad y Procesos Cognitivos, 13(1), 13-29.

Arroyave, J., Díaz, S., Toro, J., y Ceballos, G. (2007). Percepción social de los familiares de las personas que consumaron suicidio durante los años 2002-2005 en la ciudad de Santa Marta (Colombia). Duazary, 4(2), 112-118. DOI: 10.21676/ 2389783X.650. 
Baader, M. T., Urra, P. E., Millán, A. R., y Yáñez, M. L. (2011). Algunas consideraciones sobre el intento de suicidio y su enfrentamiento. Revista Médica Clínica las Condes, 22(3), 303-309. DOI: 10.1016/S0716-8640(11)70430-8.

Bahamón, M., y Alarcón-Vásquez, Y. (2018). Diseño y validación de una escala para evaluar el riesgo suicida (ERS) en adolescentes colombianos. Universitas Psychologica, 17(1), 1-15. DOI: 10.11144/Javeriana.upsy17-4.dvee

Barrios-Acosta, M., Ballesteros-Cabrera, M. D. P., ZamoraVázquez, S., Franco-Agudelo, S., Gutiérrez-Bonilla, M. L., Tatis-Amaya, J., ... y Rodríguez-Melo, C. I. (2017). Universidad y conducta suicida: respuestas y propuestas institucionales, Bogotá 2004-2014. Revista de Salud Pública, 19(2), 153-160. DOI: 10.15446/rsap.v19n2.57001

Bilbao, J., Alcocer, A., Lallemand, L., Lío, J., y Vargas, R. (2016). Reportes de suicidios en prensa de Barranquilla: un enfoque desde salud pública. CES Salud Pública, 7(2), 1-9. DOI: 10.21615/cessp.7.2.1

Brito, K., y Córdoba F. (2011). Mecanismos utilizados por sujetos que consumaron suicidio en la ciudad de Santa Marta entre los años 2004-2010. Duazary, 8(1), 20-28. doi: 10.21676/ 2389783X.1663

Builes, M., Anderson, M., Ramírez, A., y Arango, B. (2014). Suicidio en mujeres gestantes: vivencias y redes de apoyo para las familias que les sobreviven. Facultad Nacional de Salud Pública, 32(3), 332-339.

Calderón-Ramírez, V., Alcocer-Olaciregui, A., y Vargas-Moranth, R. (2017). Intentos de suicidio por intoxicación con sustancias químicas en Colombia. 2007-2013. Duazary: Revista Internacional de Ciencias de la Salud, 14(2), 149-159. DOI: 10.21676/2389783X.1963.

Campo-Arias, A., y Herazo, E. (2014). Pobreza, desigualdad y tasa de suicidio en Colombia, 2012. Duazary, 11(2), 126-130. DOI: https://doi.org/10.21676/2389783X.819

Cantú, P. (2015). Investigación en salud pública consideraciones y proceso. Cultura, Ciencia y Tecnología, 28(5), 29-36.

Cañón, S. (2011). Factores de riesgo asociados a conductas suicidas en niños y adolescentes. Archivos de Medicina, 11(1), 62-67. DOI: 10.30554/archmed.11.1.1435.2011

Cañón, S., Castaño, J., Atehortúa, B., Botero, P., García, L., Rodríguez, L., y Rincón, E. (2012). Factor de riesgo para suicidio según dos cuestionarios y factores asociados en población estudiantil de la Universidad de Manizales (Colombia), 2011. Psicología desde el Caribe, 29(3), 632-664.

Cañón, S., Castaño, J., Mosquera, A., Nieto, L. M., Orozco, M., y Giraldo, W. (2018). Propuesta de intervención educativa para la prevención de la conducta suicida en adolescentes en la ciudad de Manizales (Colombia). Diversitas: Perspectivas en Psicología, 14(1), 27-40. DOI: 10.15332/s1794-9998.2018. 0001.02

Cañón, S., Castaño, J., Muriel, L., Pérez, C., y Ramírez, S. (2016). Factor de riesgo suicida y factores asociados en reclusas de un centro penitenciario de Caldas (Colombia), 2015: Estudio de corte transversal. Archivos de medicina, 16(1), 61-73. DOI: 10.30554/archmed.16.1.1558.2016

Cañón, S., y Toro, P. (2012). Caracterización de la población con conducta suicida en la Clínica San Juan De Dios de Manizales de julio a noviembre de 2010. Archivos de Medicina (Manizales), 12(1), 83-92. Doi: 10.30554/archmed.12.1.800.2012
Cañón-Buitrago, S., Castaño-Castrillón, J., Medina-Jiménez, N., Mosquera-Escobar, K., Quintero-Cardona, C., y VaronCaicedo, N. (2017). Caracterización del intento suicida en pacientes ingresados a una institución de salud (Pereira, Colombia 2013-2014). MedUNAB, 19(3), 192-202. DOI: 10.29375/01237047.2342

Cardona, D., Medina-Pérez, O., y Cardona, D. (2016). Caracterización del suicidio en Colombia, 2000-2010. Revista Colombiana de Psiquiatría, 45(3), 170-177. DOI: 10.15446/ revfacmed.v63n3.43798

Carmona, J., Gaviria, J., y Layne, B. (2014). Suicidio, vínculos en estudiantes universitarias colombianas. Tesis Psicológica, 9(1), 102-111.

Carretero-Dios, H., y Pérez, C. (2007). Normas para el desarrollo y revisión de estudios instrumentales: consideraciones sobre la selección de tests en la investigación psicológica. International Journal of Clinical and Health Psychology, 7(3), 863-882.

Carvajal, G., y Caro, C. V. (2011). Ideación suicida en la adolescencia: una explicación desde tres de sus variables asociadas en Bogotá, 2009. Colombia Médica, 42(2), 45-56.

Castellví, P., Lucas-Romero, E., Miranda-Mendizábal, A., ParésBadell, O., Almenara, J., Alonso, I., ... y Lagares, C. (2017). Longitudinal association between self-injurious thoughts and behaviors and suicidal behavior in adolescents and young adults: A systematic review with meta-analysis. Journal of Affective Disorders, 215, 37-48. DOI: 10. 1016/j.jad.2017. 03.035

Castellví, P., Miranda-Mendizábal, A., Parés-Badell, O., Almenara, J., Alonso, I., Blasco, M. J., ... y Piqueras, J. A. (2017). Exposure to violence, a risk for suicide in youths and young adults. A meta-analysis of longitudinal studies. Acta Psychiatrica Scandinavica, 135(3), 195-211. DOI: 10.1111/ acps. 12679

Castro, V., Rangel, A., Camacho, P., y Rueda, G. (2010). Factores de riesgo y protectores para intento suicida en adultos colombianos con suicidabilidad. Revista Colombiana de Psiquiatría, 39(4), 705-715. DOI: 10.1016/S0034-7450(14) 60210-9

Ceballos, G. (2004). Características de las personas que consumaron suicidio en la ciudad de Santa Marta (Colombia) durante el año 2002: un informe de casos. Duazary, 1(1), 2428. DOI: https://doi.org/10.21676/2389783X.264

Ceballos, G., y Suarez, Y. (2012). Características de inteligencia emocional y su relación con la ideación suicida en una muestra de estudiantes de Psicología. CES Psicología, 5(2), 88-100.

Chaparro-Narváez, P., Díaz-Jiménez, D., y Castañeda-Orjuela, C. (2018). Tendencia de la mortalidad por suicidio en áreas urbanas y rurales de Colombia, 1979-2014. Biomédica, 39(2). DOI: 10.7705/biomedica.v39i3.4427

Dávila-Cervantes, C., y Pardo-Montaño, A. (2017). Impacto de factores socioeconómicos en la mortalidad por suicidios en Colombia, 2000-2013. Revista Gerencia y Politicas de Salud, 16(33), 36-51. DOI: 10.11144/Javeriana.rgps16-33.ifsm

Daza, D., Martelo, M., y Domínguez, D. (2011). Epidemiología del suicidio en la ciudad de Santa Marta (Colombia) durante los años 2004-2010. Duazary: Revista Internacional de Ciencias de la Salud, 8(2), 159-168. DOI: 10.21676/ 2389783X.212 
Daza, H., Quintero, T., y Bonilla, C. (2007). El suicidio: más que una noticia, un tema de responsabilidad social. Palabra clave, 10(2), 93-111.

Fernández-Niño, J. A., Astudillo-García, C. I., RodríguezVillamizar, L. A., y Florez-Garcia, V. A. (2018). Association between air pollution and suicide: a time series analysis in four Colombian cities. Environmental Health, 17(1), 47. DOI: 10.1186/s12940-018-0390-1

Fernández-Niño, J., Flórez-García, V., Astudillo-García, C., y Rodríguez-Villamizar, L. (2018). Weather and suicide: a decade analysis in the five largest capital cities of Colombia. International Journal of Environmental Research and Public Health, 15(7), 1313. DOI: 10.3390/ijerph15071313.

Forero, I., Siabato, E., y Salamanca, Y. (2017). Ideación suicida, funcionalidad familiar y consumo de alcohol en adolescentes de Colombia. Revista Latinoamericana de Ciencias Sociales, Niñez y Juventud, 15(1), 431-442. DOI: 10.11600/1692715x. 1512729042016.

Franco, S., Gutiérrez, M., Sarmiento, J., Cuspoca, D., Tatis, J., Castillejo, A., ... y Rodríguez, C. I. (2017). Suicidio en estudiantes universitarios en Bogotá, Colombia, 2004-2014. Ciência y Saúde Coletiva, 22, 269-278. DOI: 10.1590/141381232017221.22452015

García, J., Moncada, R., y Quintero, J. (2013). El bullying y el suicidio en el escenario universitario. Revista Colombiana de Ciencias Sociales, 4(2), 298-310. DOI: 10.21501/issn.22161201

Gonzales-Portillo, J., Gil-Arévalo, J., Hernández-Botero, D., y Henao-Sánchez, L. (2016). Evaluación de las expectativas negativas y tipo de Riesgo Suicida en estudiantes de $9^{\circ}, 10^{\circ}$ y $11^{\circ}$ de una Institución educativa del departamento del Quindío. Revista Duazary, 13(1), 7-14. DOI: 10.21676/ 2389783X.1582

González, P., Medina, Ó., y Ortiz, J. (2016). Riesgo suicida y factores asociados en estudiantes de Psicología en una Universidad pública de Colombia. Revista Habanera de Ciencias Médicas, 15(1), 136-146. DOI: 10.15446/revfacmed. v63n3.44205

Grisales, H. (2006). Usos y limitaciones de los métodos de análisis multivariados en la investigación epidemiológica. Investigaciones Andina, 8(13), 81-84.

Guerrero-Martínez, L. (2016). Descripción del intento de suicidio como constructo de violencia en la población pediátrica femenina. Revista Facultad Nacional de Salud Pública, 34(3), 306-315. DOI: 10.17533/udea.rfnsp.v34n3a05.

Hernández, R., Fernández, C., y Baptista, P. (2010). Metodología de la investigación (Vol. 3). México: McGraw-Hill.

Jaramillo, J., Escobar, A., Camacho, A., y Gonzales, D. (2015). Crisis intervention strategy for attempted suicide in children and adolescents: Interactionist perspective. CES Psicología, $8(1), 1-20$.

Jenkins, R., y Singh, B. (2000) General population strategies of suicide prevention. En K. Hawton y K. Van Heeringen (Eds.), The International Handbook of Suicide and Attempted Suicide (pp. 597-615). Chichester, UK: Wiley.

Jiménez, L. (2011). Diferencias en la conducta suicida. Estudio comparativo entre los intentos de suicidio y suicidio consumado. Tesis de doctorado no publicada, Universidad Autónoma de Madrid, Madrid, España.
Kokoulina, E., y Fernández, R. (2014). Maltrato físico y emocional en la infancia y conducta suicida en el adulto. Revista de Psicopatología y Psicología Clínica, 19(2), 93-103. DOI: 10.5944/rppc.vol.19.num.2.2014.13060

Larrotta, R., Luzardo, M., Vargas, S., y Rangel, K. (2014). Características del comportamiento suicida en cárceles de Colombia. Revista Criminalidad, 56(1), 83-95.

Loboa, N. J., y Morales, D. F. (2016). Perfil de orientación al suicidio en adolescentes escolarizados, Villahermosa-Tolima, 2013. Revista Facultad Nacional de Salud Pública, 34(1), 96104. DOI: $10.17533 /$ udea.rfnsp.v34n1a12

Luna, E., Zambrano, C., Ceballos, A., y Villalobos, F. (2017). Validación de un procedimiento estandarizado de medición de actitudes implícitas frente al suicidio. Revista CES Psicología, 10(2), 66-85. DOI: $10.21615 /$ cesp. 10.2 .5

Macana, N. (2011). Comportamiento del suicidio en Colombia, 2011. Bogotá: Instituto Nacional de Medicina Legal y Ciencias Forenses.

Manco, Y. (2015). Ser mujer y ser madre: tensiones en dos gestantes muertas por suicidio en Colombia durante el año 2010-2011. Revista de Psicología Universidad de Antioquía, 7(1), 125-142.

Manterola, C., y Otzen, T. (2014). Estudios observacionales: los diseños utilizados con mayor frecuencia en investigación clínica. International Journal of Morphology, 32(2), 634-645. DOI: 10.4067/S0717-95022014000200042

Martínez, L., Vianchá, M., Pérez, M., y Avendaño, B. (2017). Asociación entre conducta suicida y síntomas de anorexia y bulimia nerviosa en escolares de Boyacá, Colombia. Acta Colombiana de Psicología. 20(2), 189-199. DOI: http:// www. dx.doi.org/10.14718/ACP.2017.20.2.9

Massip, J., Soler, S., y Torres, R. (2011). Uso de la estadística en la Revista Cubana de Higiene y Epidemiología, 1996-2009. Revista Cubana de Higiene y Epidemiología, 49(2), 276-291.

Medina, Ó., Piernagorda, C., y Rengifo, J. (2010). Prevalencia del suicidio en adultos jóvenes en el departamento del QuindíoColombia, entre 1989 y 2008. Pensamiento psicológico, 8(15), 9-16.

Medina-Pérez, O., Ospina-Sánchez, S., y Cardona-Duque, D. (2017). Caracterización del suicidio en adolescentes del Departamento de Quindío. Colombia, 1989-2013. Revista habanera de ciencias médicas, 16(5), 784-795.

Ministerio de Salud y Protección Social (2017). Boletín de salud mental Conducta suicida Subdirección de Enfermedades No Transmisibles. Recuperado de https://www.minsalud.gov.co/ sites/rid/Lists/BibliotecaDigital/RIDE/VS/PP/ENT/boletinconducta-suicida.pdf.

Miranda-Mendizábal, A., Castellví, P., Parés-Badell, O., Almenara, J., Alonso, I., Blasco, M. J., ... \& Piqueras, J. A. (2017). Sexual orientation and suicidal behaviour in adolescents and young adults: systematic review and metaanalysis. The British Journal of Psychiatry, 211(2), 77-87. DOI: https://doi.org/10.1192/bjp.bp.116.196345.

Moher, D., Liberati, A., Tetzlaff, J., Altman, D. G. The PRISMA Group (2009). Preferred Reporting Items for Systematic Reviews and MetaAnalyses: The PRISMA Statement. PLoS Med 6(7): e1000097. DOI: https://doi.org/10.1371/journal. pmed 1000097 
Moher, D., Shamseer, L., Clarke, M., Ghersi, D., Liberati, A., Petticrew, M., Shekelle, P., Stewart, L., y PRISMA-P Group. (2015) Preferred reporting items for systematic review and meta-analysis protocols (PRISMA-P) 2015 statement. Systematic Reviews, 4(1), 1-9. DOI: 10.1186/2046-4053-4-1.

Molina, J., Gutiérrez, Y., y Oviedo, E. (2011). Depresión, autoestima y su relación con la ideación suicida en estudiantes de un colegio privado de la ciudad de Ciénaga-Magdalena (Colombia). Duazary, 8(1-Supplement), 13-19. DOI: https:// doi.org/10.21676/2389783X.1662

Montero, I., y León, O. (2002). Clasificación y descripción de las metodologías de investigación en Psicología. International journal of clinical and health psychology, 2(3).

Montoya, B (2018). Comportamiento del suicidio. Colombia, 2017. Forensis. Recuperado de http://www.medicinalegal.gov. co/documents/20143/262076/Forensis+2017+Interactivo. pdf/0a09fedb-f5e8-11f8-71ed-2d3b475e9b82.

Moreno-Montoya, J., Palacios-Espinosa, X., y Gracia-Ruiz, J. (2017). Association between religion and suicidal behaviors in cancer patients. Revista colombiana de psiquiatria, 46(4), 209-214. DOI: 10.1016/j.rcp.2016.09.007

Muñoz, E., y Gutiérrez, M. (2010). Factores de riesgo asociados al suicidio en Nariño (Colombia): estudio de casos y controles. Revista Colombiana de Psiquiatría, 39(2), 291-312. DOI: https://doi.org/ 10.1016/S0034-7450(14)60252-3

Ojeda, D., y Villalobos, F. (2011). Elementos para una Política Pública desde la Percepción del Suicidio en Nariño. Revista Colombiana de Psicología, 20(1), 57-73. DOI: https://doi.org/ $10.15446 / \mathrm{rcp}$

Oramas, J., y Santana, S. (2014). Las Revisiones Sistemáticas y la Colaboración Cochrane- Aspectos metodológicos. Educación Médica Superior, 29(2). Recuperado de http://www.ems.sld. cu/index.php/ems/article/view /540/259.

Organización Mundial de la Salud. (2014). Prevención del suicidio, un imperativo global. Recuperado de http://apps.who.int/iris/ bitstream/handle/10665 /136083/9789275318508_spa.pdf; jsessionid=5E9BD3AF25D6D3DDD30C 158C2E90111F? sequence $=1$.

Organización Mundial de la Salud. (2017). Suicidio. Nota descriptiva. Recuperado de http://www.who.int/mediacentre/ factsheets/fs398/es.

Organización Mundial de la Salud. (2018). Suicidio. Nota descriptiva. Recuperado de http://www.who.int/es/newsroom/fact-sheets/detail/suicide.

Ortega, P., Manrique, R., Tovilla, C., López, C., y Cuartas, J. (2014). Clinical and epidemiological characteristics of suicides committed in Medellin, Colombia. Revista Colombiana de Psiquiatría, 43(2), 106-112. DOI: 10.1016/j.rcp.2014.02.006

Ortegón, M., Martínez, A., y Pérez, I. (2018). Prevention versus Clinical Management of Suicide Attempts in Adolescents: What are the Costs? Revista Ciencias de la Salud, 16(2), 188202. DOI: 10.12804/revistas.urosario.edu.co/revsalud/a.6764

Óscar, A., y Beltrán. G. (2005). Revisiones sistemáticas de la literatura. Revista Colombiana de Gastroenterología, 20(1), 60-69. DOI: https://doi.org/10.1016/j.ciresp.2011.07.009

Palacio, C., García, J., Diago, J., Zapata, C., Ortiz, J., López, G., y López, M. (2005). Characteristics of people committing suicide in Medellín, Colombia. Revista de Salud Pública, 7(3), 243-25. DOI: 10.1590/s0124-00642005000300001
Palacios, X., Latiff, A., y Valderrama, P. (2006). Relación de la intención e ideación suicida con algunas variables. Revista Colombiana de Psicología, 15, 25-38. DOI: 10.15446/rcp

Palacios, X., y Ocampo, J. (2011). Situación actual del conocimiento acerca del suicidio en las personas con cáncer. Revista Ciencias de la Salud, 9(2), 173-190.

Paniagua, R., González, C., y Rueda, S. (2014). Orientación al suicidio en adolescentes en una zona de Medellín, Colombia. Revista Facultad Nacional de Salud Pública, 32(3), 314-32.

Pardo, G., y Cedeño, M. (1997). Investigación en salud, factores sociales. Bogotá, Colombia: Mc Graw-Hill-Interamericana.

Peña, P., Ortiz, Y., y Gutiérrez, M. (2009). El suicidio en Nariño: una mirada desde los observatorios del delito en cinco municipios del Departamento. Pensamiento Psicológico, 6(13), 97-198.

Pereira-Morales, A. J., Adán, A., Camargo, A., y Forero, D. (2017). Substance use and suicide risk in a sample of young Colombian adults: An exploration of psychosocial factors. The American journal on addictions, 26(4), 388-394. DOI: 10.1111/ ajad. 12552

Perestelo-Pérez, L. (2013). Standards on how to develop and report systematic reviews in psychology and health. International Journal of Clinical and Health Psychology, 13(1), 49-57. DOI: https://doi.org/10.1016/S1697-2600(13)70007-3

Pérez, A. (2014). Muertes silenciadas: Problemática del suicidio en los campesinos de La Unión (Antioquia). Revista de la Facultad Nacional de Salud Pública, 32(2), 92-102.

Pérez, C. (2010). La estadística como herramienta en la investigación psicológica. Tesis de maestría no publicada, Universidad de Granada, Granada, España.

Pérez, C., y Martín, J. (2004) Sobre los estudios longitudinales en epidemiología. Revista Española de Salud Pública, 78(2), 135-140.

Pérez, D., y Calderón, A. (2012). Prevalencia del suicidio femenino en el departamento del Magdalena (Colombia): 2004-2010. Duazary, 9(1), 87-93. DOI: https://doi.org/ 10.21676/2389783X.196

Pérez, I., Ibáñez, M., Reyes, J., Atuesta, J. y Suárez, M. (2008). Factores asociados al intento suicida e ideación suicida persistente en un centro de atención primaria. Bogotá, 20042006. Revista de Salud Pública, 10(3), 374-385. DOI: 10.1590/S0124-00642008000300002

Pérez, M., Martínez, L., Viancha, A., y Avendaño, B. (2017). Intento e ideación suicida y su asociación con el abuso sexual en adolescentes escolarizados de Boyacá - Colombia. Diversitas: Perspectivas en Psicología, 13(1), 91-101. DOI: https://doi.org/10.15332/s1794-9998.2017.0001.07

Pérez, M., Viancha, M., Martínez, L., y Salas, I. (2014). El maltrato familiar y su relación con la ideación suicida en adolescentes escolarizados de instituciones públicas y privadas de las ciudades de Tunja, Duitama y Sogamoso. Psicogente, 17(31), 80-92. DOI: https://doi.org/10.17081/psico.17.31.1471

Piedrahita, L., Paz, K., y Romero, A. (2012). Estrategia de intervención para la prevención del suicidio en adolescentes: la escuela como contexto. Revista Hacia la Promoción de la Salud, 17(2), 136-148.

Obando, D., Trujillo, Á., y Prada, M. (2018). Conducta autolesiva no suicida en adolescentes y su relación con factores personales y contextuales. Revista de Psicopatología y 
Psicología Clínica, 23(3), 198-200. DOI: 10.5944/rppc. vol.23.num.3.2018.21278

Quiceno, J., Mateus, J., Cárdenas, M., Villareal, D., y Vinaccia, S. (2013). Calidad de vida, resiliencia e ideación suicida en adolescentes víctimas de abuso sexual. Revista de Psicopatología y Psicología Clínica, 18(2), 107-117. DOI: https://doi.org/10.5944/rppc.vol.18.num.2.2013.12767

Quiceno, J., y Vinaccia, S. (2013). Calidad de vida, factores salutogénicos e ideación suicida en adolescentes. Terapia psicológica, 31(2), 263-271. DOI: https://doi.org/10.4067/ S0718-48082013000200012

Rátiva, J., Ruiz, V., y Medina, O. (2013) Análisis de las noticias sobre suicidio publicadas en un diario local del Quindío, Colombia, entre 2004 y 2011. Revista Cubana de Higiene y Epidemiología, 51(2), 184-191.

Rendón-Quintero, E., y Rodríguez-Gómez, R. (2016). Vivencias y experiencias de individuos con ideación e intento suicida. Revista Colombiana de Psiquiatría, 45(2), 92-100. DOI: 10.1016/j.rcp.2015.08.003

Reyes, H., Castro, R., Polo, L., y Ceballos, G. (2005). Características psicodemográficas de los pacientes atendidos por intento de suicidio en un hospital de tercer nivel de la ciudad de Santa Marta (Colombia). Duazary: Revista Internacional de Ciencias de la Salud, 2(1), 27-35. DOI: https://doi.org/10.21676/2389783X.281

Rodríguez, A. (2001). Autopsia Psicológica: Una herramienta útil para el peritaje psicológico. Boletín electrónico de Psicología Jurídica y Forense, volumen Mayo-Junio. [Versión electrónica]. Recuperado de http://psicologiaJuridica. org.

Rodríguez, A., Medina, O., Rozo, A., y Sánchez, S. (2013). Ideación suicida y factores asociados en un grupo de policías de Pereira-Colombia. El Ágora USB, 13(2), 411-420. DOI: $10.21500 / 16578031.110$

Rodríguez, M., Rincón, H., Velasco, M., Hernández, C., Ramos, M., y González, J. (2012). Prevalencia de trastornos mentales en una población que demanda servicios de salud en una IPS de baja complejidad y su asociación con la ideación suicida y la discapacidad percibida. Facultad Nacional de Salud Pública, 30(2), 141-151.

Rodríguez-Hernández, J., Rocha-Buelvas, A., MendietaIzquierdo, G., y Hidalgo-Troya, A. (2018). Riesgo de muerte por suicidio en población Colombiana 2000-2013. Ciência y Saúde Coletiva, 23, 3989-3996. DOI: 10.1590/1413812320182311.24812016.

Rueda, E., Díaz, P., Rangel, A., Castro, A., y Camacho, P. (2011). Diferencias de género en pacientes con suicidabilidad. Revista Colombiana de Psiquiatría, 40(4), 637-646. DOI: 10.1016/ S0034-7450(14)60197-9

Rueda, G., Rangel, A., y Camacho. (2011). Trastorno bipolar y suicidabilidad en pacientes colombianos. Revista Colombiana de Psiquiatría, 40, 108S-118S. DOI: https://doi.org/10.1016/ S0034-7450(14)60197-9

Rueda, G., Rangel, A., Castro, V., y Camacho, P. (2010). Suicidabilidad en adolescentes, una comparación con población adulta. Revista Colombiana de Psiquiatría, 39(4), 683-692. DOI: https://doi.org/10.1016/S0034-7450(14)60208-0

Salamanca, Y., y Siabato, E. (2017). Investigaciones sobre ideación suicida en Colombia, 2010-2016. Pensando Psicología, 13(21), 59-70. DOI: 10.16925/pe.v13i21.1714.
Sánchez, R., Orejarena, S., y Guzmán, Y. (2004). Características de los Suicidas en Bogotá: 1985-2000. Revista de Salud Pública. 6(3), 217-234.

Sánchez-Teruel, D., Muela-Martínez, J. A., y García-León, A. (2018). Variables de riesgo y protección relacionadas con la tentativa de suicidio. Revista de Psicopatología y Psicología Clínica, 23(3), 221-229. DOI: 10.5944/rppc.vol.23. num.3.2018.19106

Sandín, B., Valiente, R. M., Pineda, D., García-Escalera, J., y Chorot, P. (2018). Escala de Síntomas de los Trastornos de Ansiedad y Depresión (ESTAD): Datos preliminares sobre su estructura factorial y sus propiedades psicométricas. Revista de Psicopatología y Psicología Clínica, 23(3), 167-173. DOI: 0.5944/rppc.vol.23.num.3.2018.22976

Sepúlveda, R. (2008). «Vivir las ideas, idear la vida»: adversidad, suicidio y flexibilidad en el ethos de los emberá y wounaan de Riosucio, Chocó. Antípoda. Revista de Antropología y Arqueología, (6), 245-27. DOI: 10.7440/antipoda6.2008.12

Siabato, E., Forero, I., y Salamanca, Y. (2016). Asociación entre depresión e ideación suicida en un grupo de adolescentes colombianos. Pensamiento Psicológico, 15(1), 51-61. DOI: https://doi.org/10.11144/Javerianacali.PPSI15-1.ADIS.

Silva, D., Valdivia, M., Vicente, B., Arévalo, E., Dapelo, R., y Soto, C. (2017). Intento de suicidio y factores de riesgo en una muestra de adolescentes escolarizados de Chile. Revista de Psicopatología y Psicología Clínica, 22(1), 33-42. DOI: 10.5944/rppc.vol.22.num.1.2017.16170

Soriano, A. (2014). Diseño y validación de instrumentos de medición. Diá-logos, 14, 19-40. DOI: https://doi.org/10.5377/ dialogos.v0i14.2202

Suárez, E., Barrera, J., Gómez, M. T., Velásquez, N., Guzman, Y., y García, V. (2017). Cobertura periodística sobre el suicidio: ¿habría riesgo de causar efectos negativos en personas susceptibles? Persona y Bioética, 21(1), 151-160. DOI: 10.5294/pebi.2017.21.1.10.

Suárez, Y., Restrepo, D., y Caballero, C. (2016). Ideación suicida y su relación con la inteligencia emocional en universitarios colombianos. Revista Salud UIS, 48(4). DOI: 10.18273/ revsal.v48n4-2016005.

Suescùn, J., Caballero-Domínguez, C., Fuentes, C. \& Ceballos, G. (2017). Características sociodemográficas y suicidios consumados en las ciudades de Santa Marta y Bucaramanga (Colombia): un estudio retrospectivo de género 2010-2014. Cuadernos Hispanoamericanos de Psicología, 17(1), 17-28. DOI: https://doi.org/10.18270/chps..v17i1.2144

Toro-Tobar, R. A., Grajales-Giraldo, F. L., y Sarmiento-López, J. C. (2016). Riesgo suicida según la tríada cognitiva negativa, ideación, desesperanza y depresión. Aquichan, 16(4), 473486. . Doi: 10.5294/aqui.2016.16.4.6

Urrego-Mendoza, Z., Bastidas-Jacanamijoy, M., Coral-Palchucán, G., y Bastidas-Jacanamijoy L. (2017). Narrativas sobre la conducta suicida en pueblos indígenas colombianos, 19932013. Revista Facultad Nacional de Salud Pública, 35(3), 400-409. DOI: 10.17533/udea.rfnsp.v35n3a10

Vanegas, B. (2011). La investigación cualitativa: un importante abordaje del conocimiento para enfermería. Revista Colombiana de Enfermería, 6(6), 128-142. DOI: https://doi. org/10.18270/rce.v6i6.1441 
Vargas, H., y Saavedra, J. (2012). Factores asociados con la conducta suicida en adolescentes. Revista de NeuroPsiquiatría, 75(1), 19-28. DOI: https://doi.org/0.20453/ rnp.v75i1.1539

Velásquez, A., y Grisales, H. (2017). Tasa de mortalidad y años potenciales de vida perdidos por suicidio en comunas y barrios de Medellín, Colombia, 2010 y 2012. Revista Médica de Risaralda, 23(1), 8-12. DOI: 10.22517/25395203.13841

Villalobos, F. (2006). Aplicación del modelo procesual del estrés a la conducta suicida. Universidad y Salud, 1(8), 126-141.
Villalobos, F. (2009). Situación de la conducta suicida en estudiantes de colegios y universidades de San Juan de Pasto, Colombia. Salud Moental, 32(2), 165-171.

Villalobos, F. (2010). Validez y fiabilidad del Inventario de Ideación Suicida Positiva y Negativa-PANSI, en estudiantes colombianos. Universitas Psychologica, 9(2), 509-520.

Villalobos, F., Arévalo, C., y Rojas, F. (2012). Adaptación del Inventario de Resiliencia ante el Suicidio (SRI-25) en adolescentes y jóvenes de Colombia. Revista Panamericana de Salud Pública, 31, 233-239. 
